\title{
Serious Game Design by Unified Block Interactions to Support Educational Transformations
}

\author{
MARIA RENE LEDEZMA, FRANCO SIMINI \\ Núcleo de Ingeniería Biomédica (NIB), de las Facultades de Medicina e Ingeniería, Universidad de la República, Montevideo, Uruguay \\ (e-mail: rledezma@fing.edu.uy, simini@ fing.edu.uy) \\ Corresponding author: Maria Rene Ledezma (e-mail: rledezma@fing.edu.uy).
}

\begin{abstract}
: ABSTRACT This paper introduces a generalized structure for the optimal development of an adventure serious game. Present pandemic conditions induce transformations in educational methods, of which games are an attractive option. A serious game is an interdisciplinary team project for which our structure allows all members to interact irrespective of computer science proficiency. A method of interaction between unified blocks is proposed, consisting of five blocks that cover all the characteristics necessary to describe a serious game. The unified blocks are: rules, characters, scenarios, communication and score. The blocks are divided into sub-blocks that detail the characteristics of the game. The "luck" sub-block allows the real-life non-predictability dimension to be included in the game. During the interaction of the sub-blocks the different contexts of the game are created. Each context has a specific educational content goal that the player must go through. The interactions between sub-blocks are described in an XML file, common working environment for all the interdisciplinary members of the design team involves graphic designers, programmers, game designers and experts in the content to be transmitted. The principle of unified blocks is applied to the fifteen contexts of an existing game, JUSEGU, for which five new contexts are included and implemented in this paper to increase its educational content.
\end{abstract}

: KEYWORDS Serious Game; Interdisciplinary; Biomedical Engineering; Specification Tool; Project Diagram.

\section{INTRODUCTION}

A game is defined as an artificial system governed by a set of tools and rules. The player, during his/her paths must overcome a specific challenge and is expected to achieve certain goals [1]. When games are played using an electronic device such as a computer, they are called videogames [2]. Traditionally used as entertainment, this $21 \mathrm{st}$ century has seen an increase in its use for teaching, because the games can help to provide a more comprehensive teaching experience than common teaching methods [3]. Known as "Serious Games", their main goal is learning and training in specific skills [4].

Usually when a game is needed to educate students, it is better to design it from the beginning [1]. The design of serious games is usually complex as it requires the incorporation of entertaining topics with educational content. Also an important part of game development is to define clearly its mechanics as well as the learning objectives [3]. So, designing a serious game requires an interdisciplinary team that includes graphic designers, programmers, game designers and experts in the content to be transmitted $[2,5]$. Taking into account the diversity of disciplines that its design presents, where there are people with vast programming knowledge and others who do not have it, it is necessary to define "a common language" to communicate ideas and carry out the project [6]. To connect all people involved in the game design it is necessary to work on documents that reflect all the characteristics of the game. This allows the exchange of information between those involved [7, 8]. Also, having specific and well-structured documentation allows a better game maintenance and future updates [2,7].

In the present state of the art there are no design guidelines established to be followed and therefore produce efficient developments of games [9].

Nevertheless in the following sections we will see some proposals, tools and architectures used for game design. Many of the tools emphasize that the educational objective is to be clear and motivating for the player. The limits of the game must be clearly delimited, its fluidity and coherence help the player to progress towards the goal [10].

The aim of our work is to introduce a novel reusable generic methodology, the Unified Block Method (UBM), to ease the development, maintenance, and evolution of serious games in interdisciplinary settings. To this end we suggest an interaction structure between unified blocks. Each block describes a part or an aspect of the serious game involving one or more actors in its development (designers, programmers, etc.). 
The first step in game design is to select the genre in which it will be played. In the case of this work, the action / adventure genre is selected. This genre is characterized by having a protagonist who explores the game world reacting quickly to what happens on the screen [1].

The importance of the application of serious games in the teaching area has gained strength in recent years. Traditional education has its roots in solid theoretical bases and faces new challenges as continuing education is offered to a growing number of people and students, some of them remotely [11] [12]. Among the tools that have been used in recent years are serious games. Serious games can positively influence students' moods and also motivations, allowing them to learn and improve their academic performance [13]. By choosing a topic and designing the game around it, serious games allow structuring knowledge and selecting the parts to emphasize [14]. It is also possible to include within serious games some elements of real life which help to convey practical knowledge to students.

Taking into account the current situation due to the COVID 19 pandemic, the use of technological tools allows supporting distance education that is being increasingly adopted by universities and educational centers.

Knowing that serious games can contribute to education in positive ways, a Biomedical Engineering Serious Game was developed to teach electrical safety in hospitals. This game, called JUSEGU [15], is the starting point of the present initiative to generalize serious game development. By doing so we hope to obtain a methodology for efficient further serious game development

Since serious game usually includes the concept of risk, JUSEGU and our suggesting adopt it as an incentive for student learning. The student is, thus, induced to save a life or avoid an accident, and to do so he/she is forced to learn.

To carry out the design and implementation of a Serious Game, it is important to count on an interdisciplinary team prepared to cope with complex processes. For this, a unifying methodology was suggested [11]. During the development of serious games the designer should concentrate more on the game dynamics rather than on the learning content which must be clearly defined beforehand in order to produce a useful educational tool [13].

Similar features are found in several early game design architecture tools and proposals. For example, Argasinski \& Wegrzyn [5] used a system with mechanics, dynamics and aesthetics called MDA. This is a generalized game system that does not directly provide the educational elements of the game.

A generalized system focused on the design of serious games is the one proposed by Carvahlo [3] called ATMSG that describes the activities of the game, the representation of the sequences, the identification of actions, the tools and finally the objectives that the player must fulfill.

In the case of Aslan [11] in his doctoral thesis he explained a methodology that allows the complete development of educational games. GAMED (diGital educAtional gaMe development methoDology) consists of four phases. The first phase is the initial planning of the game with ideas and general design. This allows the entire interdisciplinary team to have a debate as to whether the game is feasible and what it consists of. The other three phases are standard design.

Other authors used more specific tools in game design which address: validity, resources in the game, adaptability, coherence, rules of the game based on the main objective, roles and scores [16-18].

While there are several serious game design options at the early stage of development, there is no standardized method to address it.

\section{MATERIALS AND METHODS}

Looking at the tools used in game design, we propose to include the concept of interaction between unified blocks. We suggest five blocks that cover all the characteristics necessary to describe a serious action/adventure game, from contents to appearance.

The architecture that we suggest has a great potential to be used in very complex serious games due to the simplicity of each building block. Each block is clearly defined and helps the designer to specify the system while guiding other workers in the team: graphic designers, programmers, game designers and experts in the content to be transmitted. Being a simple methodology, this also allows the system to be correctly maintained over time, as the system is created block by block.

The method is divided into three parts. The first is in charge of defining the unified blocks that contain the topics of the game. The second describes the types of relationship that these blocks have and how they interact with each other to animate the game. The third is the high-level description (XML-DTD metalanguage) of the interactions between the unified blocks.

\section{A. UNIFIED BLOCKS}

There are five unified blocks that represent the characteristics of the game: rules, characters, scenarios, communication and score. Each one admits a tree structure of sub-blocks that allow situations of great complexity while maintaining conceptual simplicity. In this way, a structure is achieved that is both complex and yet precise to adapt to the topic, see Fig. 1.

As in unified software methodologies that include interconnected blocks, we consider that the design of a serious game can be improved by insuring the reusability of its parts and by the conceptual clarity of the designs through an approach similar to the unified modeling language (UML) [19]. UML is a tool available for the programming and reuse of designs and code. Therefore, we suggest here a generalpurpose system as the basis for serious game design.

Each actor in a serious game has specific functions and related entities and is therefore entitled to a different block. The advantage of defining stereotyped blocks lies in better understanding the functions and the way they interact mutually with other blocks.

The rules block is in charge of defining the difficulty to which the player will be faced as well as the missions that the player must complete. The characters block includes the player and the extras with whom the player will interact in different ways. The scenarios block is the physical spaces where the missions will be carried out and also contains the equipment that helps to complete the missions. 


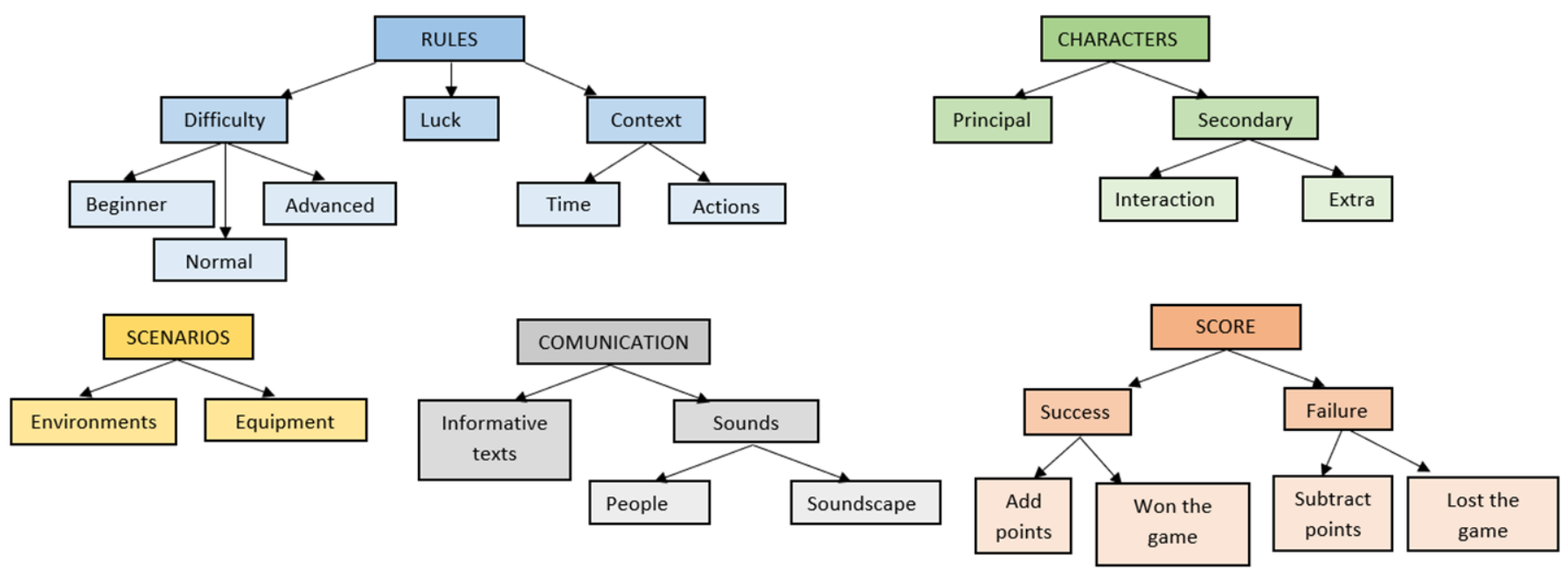

Figure 1. Unified building blocks for serious action/adventure game design.

Communication block is the important part where the designers define how the player will interact with the environment and how the educational content will be developed. Finally the score block specifies the points assigned or taken away until the player wins or loses the game.

\section{Rules}

The rule block has the task of structuring the goal that the player must seek. This block is where the educational content is located. It contains three sub-blocks:

Difficulty: determines in which level the player decides to play. This can be beginner, normal, or advanced. The selection of the difficulty determines different factors of the game increasing or decreasing its complexity.

Luck: a percentage of "luck" that the player will have is drawn, this allows the inclusion of random elements that simulate the variability and non-predictability of real life.

Context: describes the environment where the player will be, with which it must interact, where it must go, and what he/she must do to complete the goal. In addition, context defines the time the player has to complete each task and the allowed actions that the player will have in the present context.

\section{Characters}

This block represents all the characters that appear throughout the game. It contains two sub-blocks:

Principal: is the one that represents the player in the game. The player can move through different locations, interact with equipment, and complete goals in a specified time to complete the game.

Secondary: are the characters that have direct interaction with the player and those that are extras in the game to give it greater realism.

\section{Scenarios}

This block represents the locations and elements of the game that the player will interact with, towards the goal. It is divided into two sub-blocks:

Environments: places where the character moves during the assigned goal.

Equipment: elements, tools and equipment that make up the game and that the player needs to use in order to complete the goal.

\section{Communication}

This block is in charge of the general communication of the game. It is divided into two sub-blocks:

Informative texts: didactic messages that are delivered to the player through the characters or the equipment. This tool allows us to reaffirm the knowledge of the player as he completes the goal. They are in simple text format.

Sounds: produced in the environment. The sounds can be the voice of a person or the sounds emitted by the soundscape where the game takes place. They are in mp3 format.

\section{Score}

This block is in charge of establishing the player's scoring policies. It is divided into two sub-blocks:

Success: Happens when the player completes a goal. If there are pending goals, the remaining time is set, the difficulty and the primitive add points are run. The total accumulated score is thus obtained, and the game continues. If the goal is the last one, points are added up and compared to a threshold. If the accumulated points pass the threshold the player is assigned the state "won the game". But if it does not pass the threshold, the player ends the game with an insufficient score. This allows the player to complete the tasks until winning the game, confirming that he/she has been exposed to all educational content.

Failure: Happens when the player misses a goal. First the draw of luck to subtract is carried out. If the random variable is defined as "luck" in his favor, then it runs subtract points. The score varies depending on the difficulty selected.

The game ends when the player has completed all the game goals. It can also end when the player does not complete a goal and has the random variable defined as "luck" against him (bad luck) so the player is assigned the state "Lost the game"

\section{B. RELATIONSHIP BETWEEN BLOCKS}

There is a mother relationship between blocks that is typical of the game. This relationship in words would be: "The principal character fulfills certain rules of action in a given scenario exchanging communication, obtaining score points".

In this formal schematization of an adventure serious 
game we can consider a game as the implementation of the relationships between the five defined blocks. All games can be specified by relationships between blocks. Each game is characterized by a set of relationships that operate on subblocks. The definition of sub-blocks (principal character, extra character, etc.) is intrinsic to each game and gives it some of its unique characteristics. The relationships between the blocks constitute a high level of game specification. Encapsulating the details themselves that make up the generic relationship between specific sub-blocks, this characterization of a serious adventure game is the construction of a "block by block" game. For this, it is necessary to define "contexts lists" based on sub-blocks that allow the construction of each context. In this way, a detailed specification tool is proposed, accessible by all members of the interdisciplinary team.
For example: The player moves in the contexts of the operating room (OR) where the doctor tells him to calibrate the anesthesia machine before the scheduled surgery. During the procedure a person is observed carrying surgical material for surgery, if the player leaves aside his assigned task to interact with the irrelevant transfer of material, the player will lose many points or, in the worst case, the game all together. But if the player focuses on the set goal, he/she will score points. Therefore, in the list of contexts, the main goal must be the first structured item to be defined using several elements of the sub-blocks. Then to give realism to the game, other elements are generally used to take into account the relationship between the sub-blocks.

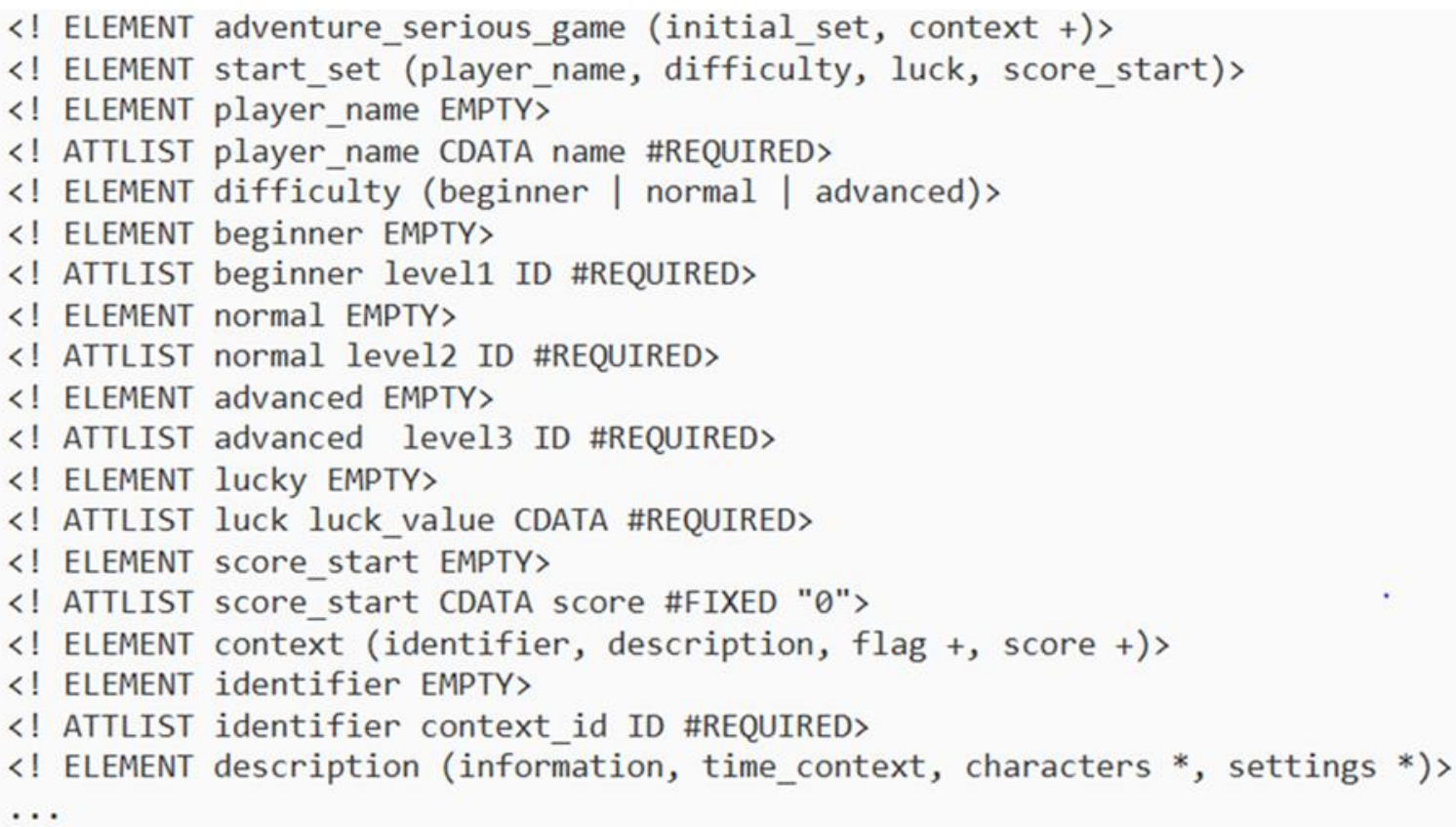

Figure 2. DTD file fragment that structures a serious adventure game

\section{LANGUAGE FOR DESCRIBING INTERACTIONS BETWEEN BLOCKS}

With the blocks and sub-blocks defined, it is necessary to express the interactions they present in simple language for the entire interdisciplinary team. The language must be able to describe the goals, the characters, and the settings. For this, the language that best adapts and can synthesize the content of the game is sought.

A language that adapts to legible documentation due to its hierarchical structure and the possibility of association between different tags is the XML extensible markup language [20]. In the literature, the XML language has been used for the description of games [7, 21, 22]. Using the XML language, the format chosen to describe the interaction between blocks is the DTD (document type definition) since it is possible to describe, restrict and verify structures in a document, in addition to being able to make declarations of various elements [23]. It is the simplest of all XML schemas [24], Therefore, the entire interdisciplinary team can understand it even if its members have no advanced programming skills.

The DTD file is mainly made up of ATTLIST and
ELEMENTS. As there are only two entities that structure the XML file, it greatly facilitates the hierarchical structure of the blocks and the definition of each element.

By having the DTD file structured, it is possible to fill each element in the XML file. This allows us to describe a serious game based on the five UBM blocks. In case the interdisciplinary design group has not placed a mandatory element, an error will be shown. This allows any member of the team to fill in the structure.

\section{RESULTS}

\section{A. XML STRUCTURE OF THE GAME}

The structure of the file that defines the adventure serious game (Figure 2) is composed of the initial configuration and the context.

Initial configuration: is in charge of selecting the player's name, the game's difficulty, defining the value of luck and setting the global score at 0 .

Context: as shown in Figure 2, it may appear one or more times. Each context describes a goal that the player must meet to win the game. Each context consists of an identifier, a description, a flag and finally the score 
- Identifier is unique to each context. This allows it to be identified in the context list. With this identification, the game can be generated in order or in a random way, giving the game more dynamism and preventing the game from being predictable.

- Description: the description is made up of four elements that are:

- Information: Indicates the goal that must be completed and the steps to be taken. The information is created here during the development phase of the game by the educational content expert.

- Time: specifies the time that the player has to complete the goal. This depends upon the difficulty assigned to the game.

- Characters: contains the principal character and secondary characters for interaction and extra characters. The contents author defines the characteristics of these characters, the role they play according to the information, the location, the actions allowed by each character and the type of

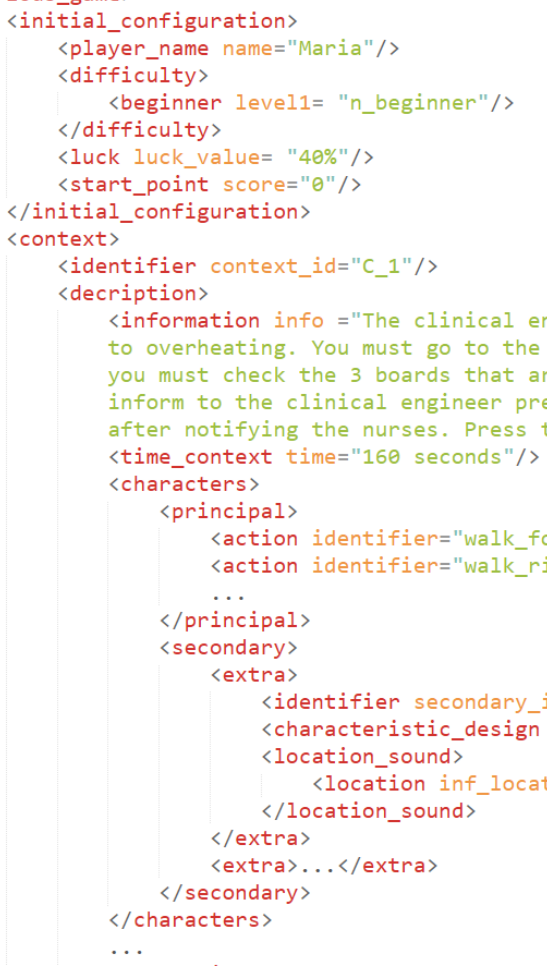

Figure 3. Unified blocks application example fragment in "JUSEGU". This context deals with the verification of the electric distribution board

\section{B. IMPLEMENTATION OF UBM IN JUSEGU}

We use UBM to describe the 15 existing contexts in JUSEGU [15]. The descriptions were correct keeping the game information for future maintenance. Likewise, UBM was implemented in 5 new contexts for the game. In Figure 3 the application of one of these contexts can be seen: the verification of the electrical distribution board. Figure 4 shows the implementation of the new context in the game.

The five new proposed contexts are:

Ground impedance measurement: This context allows the player to contact the maintenance manager of the hospital and to use a tellurometer to measure the grounding impedance of the hospital. If the grounding is incorrectly placed, the player can correct it, avoiding risks of ill grounding in case of equipment failure. communication they will have with the player, whether by text or by sounds. Each character contains a unique identifier to recognize it.

- Scenarios: Spaces where the contexts will take place. All the environments in which the player will interact to complete the goal are specified. The equipment found in each environment and the specific equipment with which the player will interact is also specified. A unique identifier is assigned to each environment and each piece of equipment, with a design description and a specific location. Likewise, some rooms and facilities include specific soundscapes and informative text.

- Flag: A flag is defined for each context. The flag indicates whether the context goal was completed or not, to define the score.

- Score: First the state of the flag is checked to see if the goal has been completed. For this, it is defined whether it is success or failure (see sub-block A.5 "Score" in Materials and Methods).

Electric distribution board overheating: In this context, the player receives a message from the clinical engineering department that one of the hospital boards is overheated. As a consequence, the player must pick up an infrared temperature measurement device to identify the faulty board, Figure 4 .

Careless student: In this context an intern student is helping the radiologist and forgot to place the gonad protector on the patient before taking a pelvic X-ray. The player must inform the student to put the protector on the patient before the radiologist arrives in the room.

Power failure during surgery: In this context, the player is notified that an electrosurgical unit failed and he/she must go to the operating room " $\mathrm{A}$ " to pick it up. The player then goes to the clinical engineering department to perform the test with the electrosurgical tester to find out the failure to fix. 
New medical intern student: In this context, a new medical intern is introduced to the hospital. The medical intern is not sure how to use a vital signs monitor, so the player must explain how to use the equipment and must also help him to filter out noise from an electrocardiogram.

\section{CONCLUSIONS}

The proposed methodology UBM generalizes an action/adventure serious game. This allows describing its characteristics so that the entire interdisciplinary design team can understand it, making it easier for development and future maintenance.

With a "mother interaction" that structures each goal of the game in five unified blocks -which in turn are branched

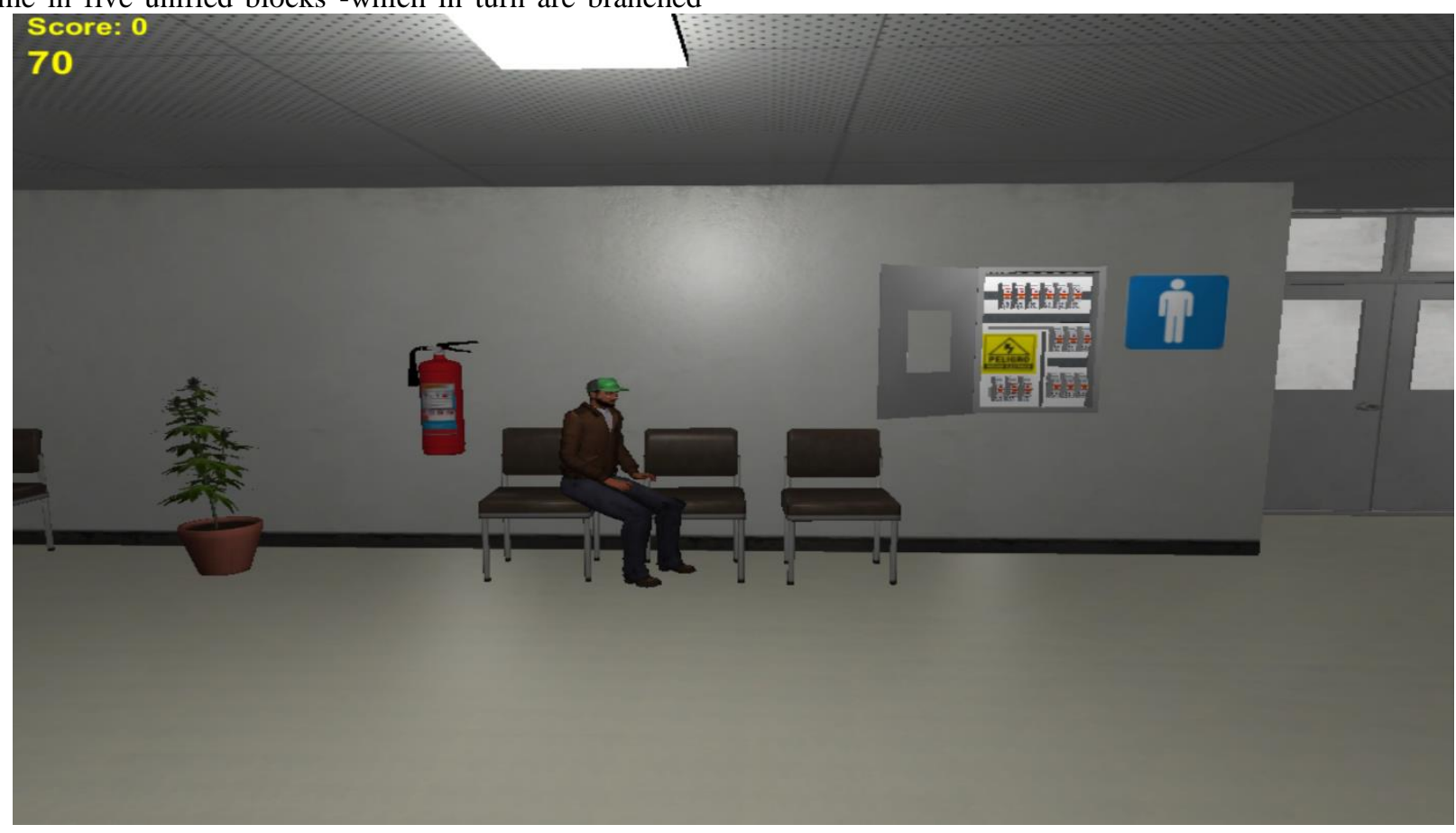

Fig 4. Implementation of a new context in JUSEGU: Verification of the electric distribution board overheating.

Our UBM method was applied to the 15 contexts of JUSEGU, confirming its optimal description and was also used to add five new contexts with this format.

We have tested this block approach with JUSEGU and we intend to do the same in the future for other serious games such as SERVOGLU [25], SIMVENT-DOCEO [26] and SEPEPE [27].

Unlike medical images with DICOM and electronic clinical records with CDA format files, there are no accepted standards to specify and design serious games. Following the interesting proposal of Moreno [7] we introduce in UBM a simple syntax and clear entities to efficiently tackle serious game specification and design.

The last few years have seen an increase in the use of games in Medicine. This helps students to learn [28]. There are games that deal with medical education that in the long run will save lives. To ensure this it is necessary to correctly define their objectives. For example EMERGE is a game used by 5 th to 12 th semester students at Cologne University [29]. This game allows improving and strengthening the skills of students by solving standard surgical protocols.

With UBM we innovate by introducing a standardization of action / adventure games, to help design and implement into sub-blocks- the necessary generalization is obtained for efficient interdisciplinary work. This methodology also allows a complete and well-guided documentation.

The concept of "luck" adopted from JUSEGU allows us to include unpredictability in different contexts. This acts in favor or against the player, just as it happens in real life.

The generation of the DTD file, structured as elements and attributes, validates XML file syntax. It generates a simple, hierarchical file that any member of the interdisciplinary team can contribute with authorship. This allows for optimal communication between all team members in a collaborative and cooperating manner. 
https://doi.org/10.1016/j.future.2018.06.013

[6] M, Zhu, A.I. Wang "Model-driven game development: A literature review," ACM Computing Surveys, vol. 52, issue 6, pp. 1-32, 2019. https://doi.org/10.1145/3365000.

[7] P. Moreno-Ger, J. L. Sierra, I. Martínez-Ortiz, B. Fernández-Manjón, "A documental approach to adventure game development," Science of Computer Programming, vol. 67, issue 1, pp. 3-31, 2007. https://doi.org/10.1016/j.scico.2006.07.003.

[8] S. Tang, M. Hanneghan, "Game content model: an ontology for documenting serious game design," In Proceedings of the 2011 Developments in E-systems Engineering, 2011, pp. 431-436. https://doi.org/10.1109/DeSE.2011.68.

[9] M. Kosa, M. Yilmaz, R. V. O'Connor, and P. M. Clarke, "Software engineering education and games: A systematic literature review," $J$. Univers. Comput. Sci., vol. 22, no. 12, pp. 1558-1574, 2016.

[10] J. Cain and P. Piascik, "Are serious games a good strategy for pharmacy education?," Am. J. Pharm. Educ., vol. 79, no. 4, 2015. https://doi.org/10.5688/ajpe79447.

[11] S. Aslan, Digital Educational Games: Methodologies for Development and Software Quality, PhD Thesis, Virginia, 2016, pp. 20-72.

[12] J. E. Monzon, "The challenges of biomedical engineering education in Latin America," in Proceedings of the IEEE Engineering in Medicine and Biology 27th Annual Conference, Shanghai, China, September 1-4, 2005 pp. 2403-2405. https://doi.org/10.1109/IEMBS.2005.1616952.

[13] Y. Zhonggen, "A meta-analysis of use of serious games in education over a decade," Int. J. Comput. Games Technol., vol. 2019, no. 3, 2019. https://doi.org/10.1155/2019/4797032.

[14] E. Boyle, et al., "An update to the systematic literature review of empirical evidence of the impacts and outcomes of computer games and serious games," Computers \& Education, vol. 94, pp. 178-192, 2016. https://doi.org/10.1016/j.compedu.2015.11.003.

[15] E. Marichal, F. Simini, JUSEGU: Juego de seguridad eleéctrica en hospitales in. Universidade FEEVALE, Feira de Iniciação Científica, Novo Hamburgo, 2015, pp. 303-328.

[16] G. Ben-Sadoun, V. Manera, J. Alvarez, G. Sacco, and P. Robert, "Recommendations for the design of serious games in neurodegenerative diseases," Front. Aging Neurosci., vol. 10, no. FEB, pp. 1-7, 2018. https://doi.org/10.3389/fnagi.2018.00013.

[17] M. Graafland et al., "How to systematically assess serious games applied to health care," JMIR Serious Games, vol. 2, no. 2, 2014. https://doi.org/10.2196/games.3825.

[18] D. Buchinger and M. da Silva Hounsell, "Guidelines for designing and using collaborative-competitive serious games," Comput. Educ., vol. 118, no. February 2017, pp. 133-149, 2018. https://doi.org/10.1016/j.compedu.2017.11.007.

[19] R.P. De Lope,N. Medina-Medina, "Using UML to model educational games," in Proceedings of the 8th International Conference on Games and Virtual Worlds for Serious Applications (VS-GAMES), Barcelona, Spain, September 7-9, 2016, pp 1-4. https://doi.org/10.1109/VSGAMES.2016.7590373.

[20] W3C, XML Technology-Schema, 2015, [Online]. Available at: https://www.w3.org/standards/xml/schema

[21] J. R Quiñones, A. J. Fernández-Leiva, "XML-based video game description language," IEEE Access, vol. 8, pp. 4679-4692, 2019. https://doi.org/10.1109/ACCESS.2019.2962969.

[22] W. Mestadi, K. Nafil, R. Touahni, and R. Messoussi, "An assessment of serious games technology: toward an architecture for serious games design,” Int. J. Comput. Games Technol., vol. 2018, 9834565, 2018. https://doi.org/10.1155/2018/9834565.

[23] A. Skonnard, M. Gudgin, Essential XML Quick Reference, AddisonWesley, Indianapolis, 2002, 15-33 p.

[24] D. Lee, W.W Chu, "Comparative analysis of six XML schema languages," ACM Sigmod Record, vol. 29, issue 3, pp. 76-87, 2000. https://doi.org/10.1145/362084.362140.

[25] G. Ashby, I. Ferrer, \& F. Simini, "SERVOGLU: simulador gráfico de sistemas fisiológicos basados en ecuaciones diferenciales para enseñanza y ensayo de conductas clínicas," in XXI Congreso Argentino de Bioingeniería X Jornada de Ingeniería Clínica, Cordoba, Argentina, October 25-27, 2017. (in Spanish)

[26] F. Simini, et al., "SIMVENT-patient simulator to test mechanical ventilators," in Proceedings of the VI Latin American Congress on Biomedical Engineering CLAIB'2014, Paraná, Argentina, October 29. 31, 2014, pp. 71-74. https://doi.org/10.1007/978-3-319-13117-7_19.

[27] M. Alzugaray, C. Vega, S. Vico, "SEPEPE Seguimiento Perinatal Personalizado. Customized Perinatal (Thesis). Montevideo, 2021. (in Spanish)

[28] R. Wang, S. DeMaria, A. Goldberg, and D. Katz, "A systematic review of serious games in training: Health care professionals," Simul. Healthc., vol. 11, no. 1, pp. 41-51, 2016 https://doi.org/10.1097/SIH.0000000000000118.

[29] S. H. Chon et al., "Serious games in surgical medical education: A virtual emergency department as a tool for teaching clinical reasoning to medical students," JMIR Serious Games, vol. 7, no. 1, pp. 1-11, 2019. https://doi.org/10.2196/13028.

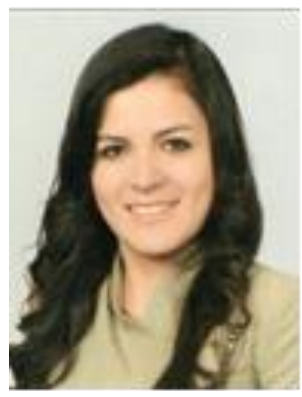

MARIA RENE LEDEZMA is a Biomedical Engineer graduated from the Faculty of Informatics and Electronics of the Universidad del Valle, Cochabamba, Bolivia. Student of a Master's degree at the Faculty of Engineering of the Universidad de la República Montevideo, Uruguay. Her Master's Thesis refers to knee biomechanics automatic detection during motor tasks.

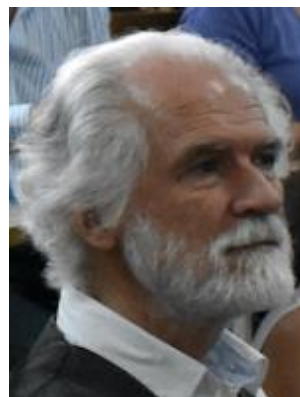

FRANCO SIMINI is a Professor of Biomedical Engineering and Medical Informatics, Universidad de la República, Uruguay. Founder of the Núcleo de Ingeniería Biomédica (NIB) at the University Hospital. Research on computer / mobile equipment for clinical use. Courses, (undergraduate \& graduate), patents, publications and technology transfer. 